\title{
LINEARIZATION OF THE FERMILAB RECYCLER HIGH LEVEL RF*
}

\author{
J. Dey", S. Dris, T. Kubicki, J. Reid, FNAL, Batavia, IL 60510, USA
}

\section{Abstract}

In studying the Recycler high level RF, it was found that at $89 \mathrm{kHz}$, the lowest frequency required by the system, some nonlinearities in magnitude and phase were discovered. The visible evidence of this was that beam injected in a barrier bucket had a definite slope at the top. Using a network analyzer, the S-parameter $S_{21}$ was realized for the overall system and from mathematical modeling a second order numerator and denominator transfer function was found. The inverse of this transfer function gives our linearization transfer function. The linearization transfer function was realized in hardware by summing a high pass, band pass and low pass filter together. The resulting magnitude and phase plots, along with actual beam response will be shown.

\section{INTRODUCTION}

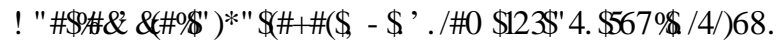
9\%: 7\&)8*\$4\$6/4(\$65\$; <<<9\#4=\$4\&\&\#(\#\%/) 8*\$+6(/.\$\$? 4\&" ./4/)68\$68. ). / \$65\$4@ @4: @8: \$AB\$)(6C4//\$ - \$2<\$EF GH

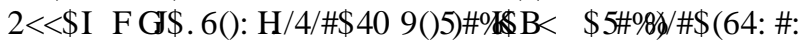

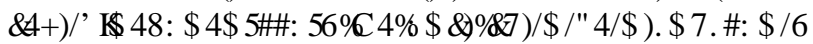
860 9\#8.4/\#\$56\$/"\#\$: \%669)8: 78\#: \$@ \$/"\#\$L M\$679(\#:

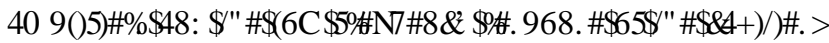

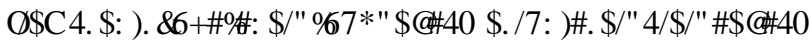
9\%5)(\#\$\#")@/\#: \$4\$. (69\#\$5\% \$78\#N74(\$ @\#40 \$\&" 4\%\#

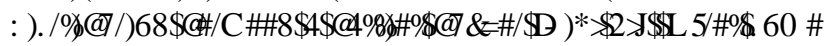

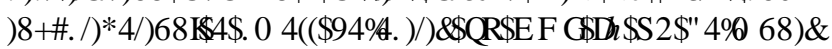
860 968\#8/J\$ )87.6): 4(\$\$60 968\#8/\$C4. \$5678: \$)0 96.\#: \$68

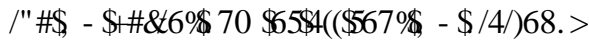

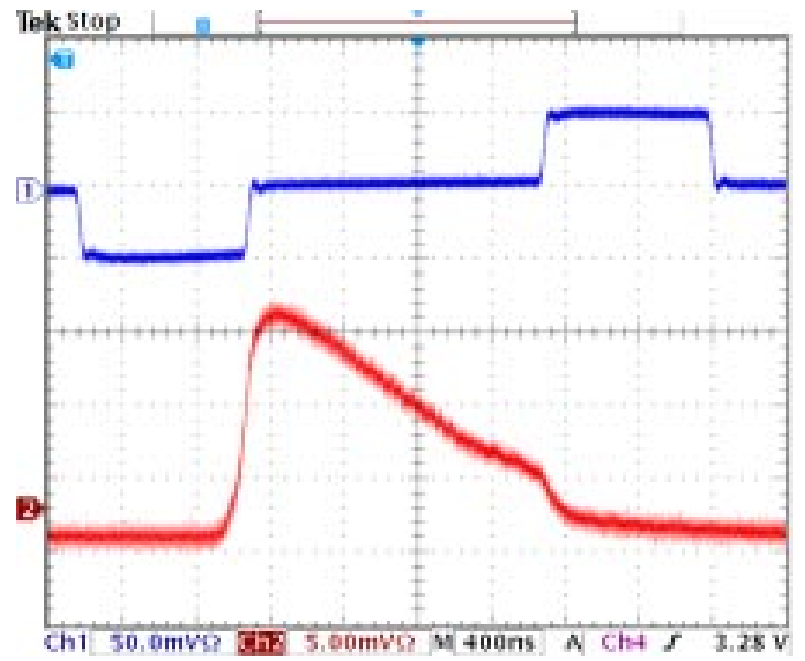

Figure 1: Recycler Barrier Bucket (blue) and Beam Profile (red) before Compensation

TTTTTTTTTTTTTTTTTTTT

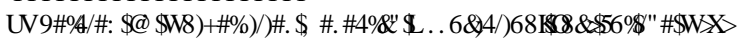

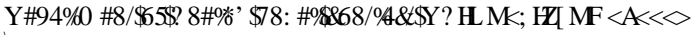
':\#'] $584\left({ }^{*} 6+\right.$

\section{LINEARIZATION}

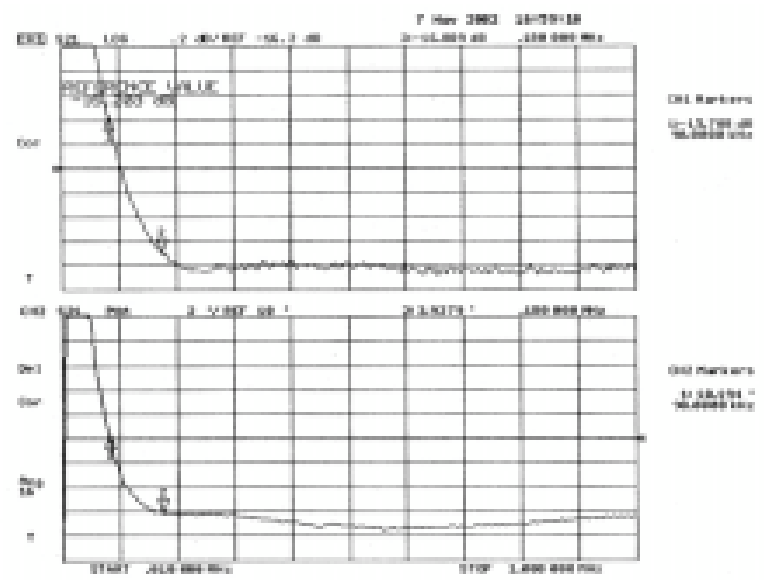

Figure 2: Nonlinear Region of the Recycler High Level RF

Using an Agilent 8753 ES Network Analyzer, the system's frequency response was measured using the vector $R F$ sum signal from four cavities and is shown in Figure 2. The nonlinearity in magnitude and phase was found to be $1.1 \mathrm{~dB}$ and $6.26^{\circ}$ in phase from $90 \mathrm{kHz}$ to 180 $\mathrm{kHz}$. Most of the error in flatness is due to the Amplifier Research 3500A100 Broadband RF Amplifier. The goal was to linearize the frequency in the region from $90 \mathrm{kHz}$ to $1 \mathrm{MHz}$ with a flatness in amplitude of $0.26 \mathrm{~dB}$ and $1.8^{\circ}$ in phase. Using the program Filter Light ${ }^{\circledR}$ 2.1, we were able to quickly realize that a second order high pass Chebyshev 1 Filter (Eq. 1) was a close approximation to the transfer function shown in Figure 2.

$$
\frac{s^{2}}{s^{2}+9.7 \times 10^{4} s+4.5 \times 10^{10}}
$$

Taking the inverse of transfer function Equation 1 yields transfer function Equation 2, a good starting point in creating a compensation network that would flatten out the low-end response.

$$
\frac{s^{2}+9.7 \times 10^{4} s+4.5 \times 10^{10}}{s^{2}}
$$




\section{Error-based Optimization}

Taking the $801 \mathrm{~S}_{21}$ points $(10 \mathrm{kHz}$ to $999.6 \mathrm{kHz})$ from the Network Analyzer and multiplying them by the linearizing function for each frequency, gives the compensated response. These results were analyzed at $90 \mathrm{kHz}$ intervals from $90 \mathrm{kHz}$ to $990 \mathrm{kHz}$ to find the smallest root mean square percentage error. By iteratively making changes to the linearizing function, a new linearizing function (Eq.\$3) was found that gave the smallest compromise in magnitude and phase for the root mean square percentage error.

$$
\frac{s^{2}+1.8 \times 10^{5} s+6.2 \times 10^{10}}{s^{2}+1.26 \times 10^{5} s+4 \times 10^{9}}
$$

By breaking the transfer function (Eq. 3) into three parts, using the same common denominator, three filters could be constructed and summed together. The $\mathrm{s}^{2}$ term in the numerator is a Second Order High Pass Bessel Filter (Fig. 3.) The s term in the numerator is a First Order Band Pass Butterworth Filter (Fig. 4.) The constant term in the numerator is a Second Order Low Pass Matched Filter (Fig. 5.)

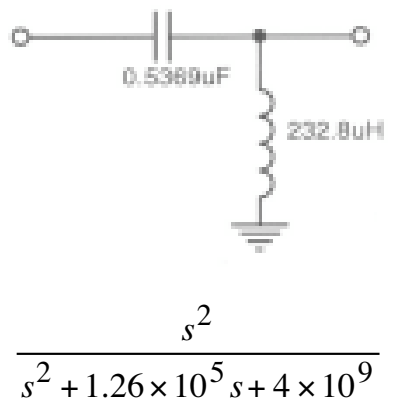

Figure\$:\$Second\$Order\$erm\$ilter

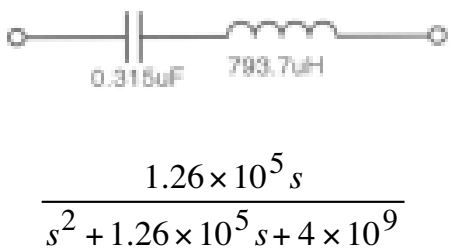

Figure\$1:First\$Order\$Term\$ilter

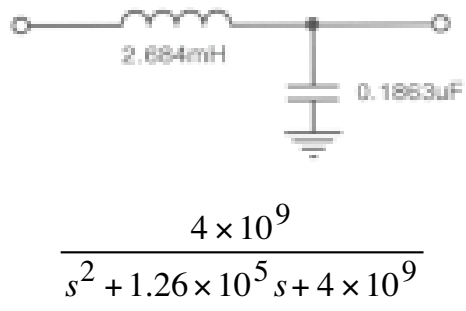

Figure\$5:\$Constant\$Term\$ilter

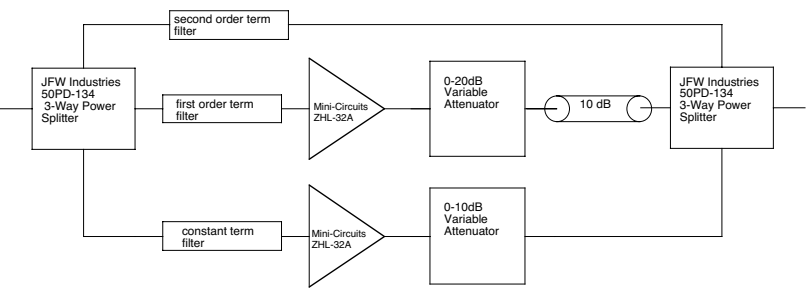

Figure 6: Block Diagram of Linearizing Circuit

\section{IMPLEMENTATION}

The filter program was used to implement the individual parts of the transfer function. Each section was found by applying the appropriate filter type that would implement each numerator term. Once this was found, the filter program allowed the user to change the values of the capacitors and inductors. The program has the capability of generating a new transfer function from these values of components. This transfer function was compared to the theoretical model. It was necessary to find three separate functions that implemented the numerator term over a common denominator. Coefficients in front of the different terms were corrected by the appropriate amount of gain (Fig. 6.)

The theoretical values of the phase obtained from the mathematical modeling of each part of the compensation transfer function were used as a basis to compare how well the real circuit approximates the ideal circuit. The phase component of each part was calculated at $89 \mathrm{kHz}$ and up to the $11^{\text {th }}$ harmonic. Measurements of each part of the compensated transfer function's phase were compared to the ideal phase results. The capacitor and inductor component values were changed to better approximate the ideal case for each part. This iterative process was used until the best approximation of the ideal transfer function was found for each case. The circuits were built using surface-mounted capacitors and inductors. Using surface-mounted parts enabled a closer approximation of ideal component values, since parasitic lead inductances are much less.

The construction of the compensation circuit required the use of two 3-way resistive power dividers/combiners, amplifiers, fixed value and variable attenuators, and the filter circuits to realize the function. The second order high pass filter for the $\mathrm{s}^{2}$ term requires no gain adjust. The First Order Band Pass Butterworth filter required 3.1 dB of gain to realize the s-term part of the compensated transfer function. The Second Order Low Pass Matched filter required $23.8 \mathrm{~dB}$ of gain to realize the constant term of the compensated transfer function. The amplifiers used were Mini-circuits ZHL-32A with $25 \mathrm{~dB}$ of gain. Variable attenuators were placed at the output of both amplifiers, while an additional $10 \mathrm{~dB}$ attenuator was placed with the amplifier and variable attenuator that controlled the gain for the s-term of the compensated transfer function. 

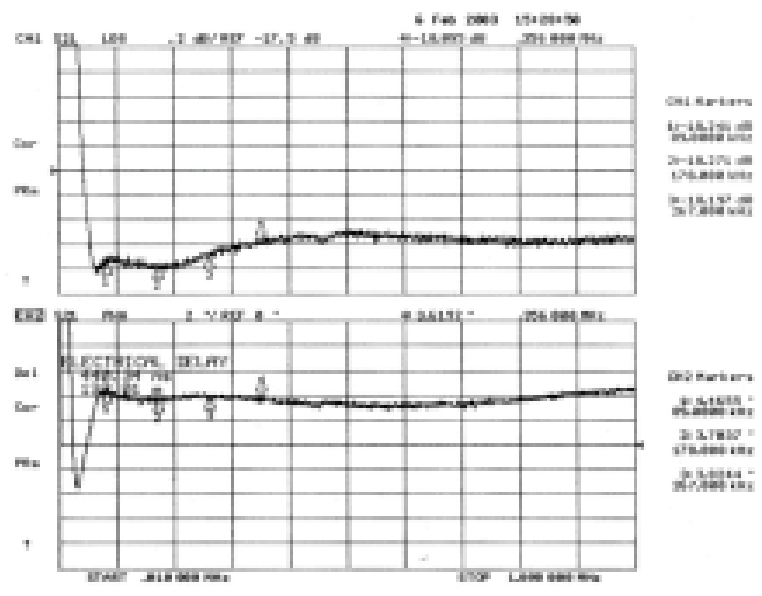

Figure 7: System's Response after Compensation

Phase matching of each section is very important to ensure that the individual transfer functions add up algebraically. Otherwise, the compensation transfer function will be a vector sum of all the components, and this would not realize the correct compensation transfer function. Figure 7 is the final compensated system response using the new linearizing circuit. The improvement in magnitude and phase at $90 \mathrm{kHz}$ is readily apparent when compared to Figure 2.

\section{CONCLUSION}

Final adjustments to the system were made with beam (Fig. 8) in the Recycler. While looking at the beam in the Recycler, the slope in the beam profile can be corrected by using the variable attenuators. The variable attenuators provide the flexibility to simultaneously compensate for the magnitude and phase response that will ensure a linear beam profile. In the event that components in the high level RF Recycler system need to be changed, the compensation network can be quickly adjusted to account for any differences. Long-term stability looks good, as no major adjustments have been necessary to date. Further operational experience will determine long-term stability.

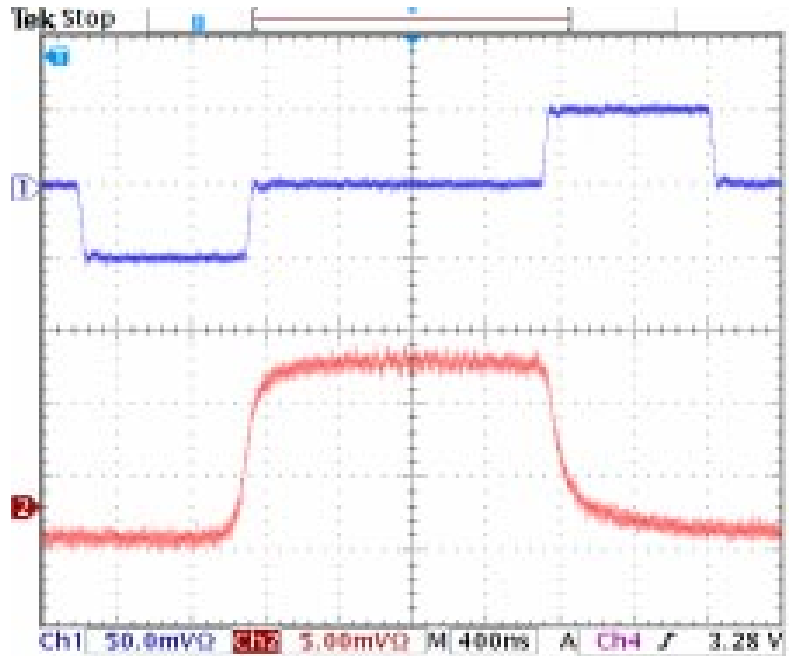

Figure 8: Rec' cler Barrier Bucket (blue) and Beam Profile (red) after Compensation

\section{REFERENCES}

[1] J. E. De', D. W. Wildman, "Wideband RF S' stem for the Fermilab Rec' cler Ring," 1999 PAC, p. 869, New York Cit', March 1999. 